Notas técnicas 



\title{
TECNOLOGÍA/INGENIERÍA: UN RESUMEN DE CONCEPTOS
}

Rodolfo Herrera J.

\author{
"Solo tiene significación lo general. \\ General equivale a dinámico, relacional, referencial, integral”. \\ Luis Lara Saborío.
}

\begin{abstract}
Resumen
Este breve ensayo tiene por objeto resumir algunos conceptos sobre tecnología-ingeniería, que sirvan para aclarar algunas de las nociones básicas respecto a este tema, y los cambios conceptuales producidos por el surgimiento de la civilización industrial y la cultura moderna.
\end{abstract}

Palabras clave: tecnología-ingeniería, técnica, definiciones, ciencia-tecnológica, diseño, práctica, artefacto, proceso, predicción-tecnológica, dirección/acción, clasificación de tecnologías.

\begin{abstract}
This brief essay has the main purpose of summarizing some concepts about technology/engineering with the purpose that they may clear some of the basic notions with respect to the topic. This work also considers conceptual changes due to the rise of the industrial civilization and modern culture.
\end{abstract}

Key words: Engineer-Technology, technique, definitions, science-technology, design, practice, device, process, prediction-technology, direction/action, classification of technologies.

\section{INTRODUCCIÓN11.}

El análisis de la literatura sobre lo que hoy se ha llamado el "fenómeno tecnológico" es muy extenso, lleno de enfoques parciales, inquietudes sociales y, en muchos casos, con planteamientos que no contribuyen teóricamente en nada y que producen confusión conceptual. El rango de los enfoques e interpretaciones produce una extensión del concepto muy amplia, que corre desde los extremos de "cosa material" hasta de "valor social". Especialmente cuando se trata sobre las implicaciones sociales y el debate sobre el valor de la tecnología, ya que la necesidad de una mayor claridad conceptual se convierte en una tarea no estéril.
A continuación se da una lista de interpretaciones del término tecnología, la cual seguramente no agota a todo el conjunto de elementos de su extensión lógica:

- Como objeto concreto: objeto tecnológico concreto o artefacto (pe. instrumento, maquinaria, equipo, computadora, hardware, mercancía).

- Como objeto conceptual: cuerpo de conocimiento, sistema conceptual, sistema de información, modelo, plan, proyecto, diseño, patente.

- Como ciencia: ciencia aplicada, conocimiento aplicado, saber, saber-como, conocimiento práctico, aplicación o la organización del conocimiento para propósitos prácticos, aplicación de la ciencia al proceso productivo. 
- Como método: conjunto de reglas, conglomerado de técnicas, saber hacer cualquier cosa. Como cultura: componente del sistema cultural de una sociedad, subsistema conceptual de la cultura

- Como práctica social: actividad, actividad orientada empírico-transformadora, actividad teórica-conceptual, actividad cultural, actividad científica, modalidad de la actividad práctica, arte, conjunto de prácticas.

- Como sistema social concreto: sistema de control racionalizado, sistema de manipulación sobre los hombres, sociedad tecnológica, mundo artificial, institución.

- Como estructura social: modo de organización, configuración de relaciones, orden tecnológico, medio específico artificial (no natural).

- Como capacidad humana: habilidad, destreza de montaje (construcción, elaboración), creatividad práctica para la innovación, destreza de operación, (manejo, funcionamiento de tecnología blanda), capacidad para la acción o decisión, saber-hacer.

- Como capacidad o fuerza social: medio de producción concreta o conceptual, totalidad de los medios, herramientas, potencia e información orientada, uso de funciones de producción, fuerza productiva, modo de organización.

- Como proceso: proceso productivo, cambio, evolución, aplicación de energía.

- Como filosofía: filosofía moral, discurso o conversación sobre la práctica y las artes, teoría de la práctica, estudio sistemático.

- Como sistema de enseñanza: lo que tiene relación con una disciplina de ingeniería (especialmente no social).

- Filológicamente, el término es una combinación del griego techne, "arte de", con logos, palabra, conversación; entendiéndose en Grecia como un discurso sobre las artes, tanto finas como aplicadas. El término en inglés aparece en el Siglo XVII, usado sólo para entender una discusión sobre las artes aplicadas y gradualmente estas "artes" llegaron a ser el objeto de la designación.

Lo más corriente es la identificación del concepto con sólo uno de los términos de su extensión. Así por ejemplo, la interpretación más frecuente en la conversación o en el discurso popular es la de cosa concreta o artefacto (máquina, instrumento, equipo). Esta reducción de un concepto (de lo abstracto) a sustancia, se extiende casi a todo nivel cultural, utilizándose el concepto de ciencia y de tecnología, o ambos, como cosas concretas. El otro extremo es el de considerar a la tecnología como "ciencia aplicada", lo cual se analizará también en este ensayo.

Se presenta un resumen dedicado a la definición y aclaración de las nociones de tecnología o ingeniería, surgidos en la civilización industrial y la cultura moderna.

\section{TECNOLOGÍA O INGENIERÍA²}

Práctica $^{3}$ social (actividad social orientada) fundamentada en el conocimiento científico, concerniente con: (i) el diseño de artefactos y procesos (naturales o sociales) y de la organización, normalización y el planeamiento de las acciones humanas; (ii) la dirección/acción de la producción, el control ${ }^{4}$, la reparación y el mantenimiento de los artefactos o procesos (naturales 0 sociales).

La "actividad orientada" es un tipo de actividad humana deliberada o intencional, dirigida a lograr una finalidad determinada o meta, fundamentada en el conocimiento científico y por tanto consiste de un conjunto acciones racionales.

Esta definición general contiene tres conceptos básicos: [actividad, conocimiento, artefacto], necesarios para la comprensión del fenómeno tecnológico de nuestro tiempo. A continuación, se darán breves explicaciones de estos elementos conceptuales.

\section{ARTEFACTO.}

Es un objeto "hecho por el hombre", sea una cosa o sistema artificial: material o conceptual, o un proceso material de un sistema $^{5}$, que produzca el cambio de un estado natural o social.

La definición amplía el concepto común de "artefacto" como un objeto material particular (telé- 
fono, máquina, etc.), ampliando la extensión lógica a un conjunto que contiene entidades conceptuales (pe., planes, reglas, etc.) y transformaciones humanas y del entorno no-humano (pe., una ciudad o un sistema social o el estado de sus componentes, etc.). Una tipología general de éstos es la siguiente:

- Artefactos materiales sean inanimados (máquinas, fábricas, un hardware); vivientes (un cultivo o ganado) o sociales (una organización, un proceso social).

- Procesos o sistemas de procesos materiales (procesos industriales, organizaciones sociales, movimientos sociales, etc.).

- Artefactos conceptuales (símbolos, software, teorías, reglas, etc.).

Como son sistemas, tienen un mecanismo o conjunto de procesos (o secuencias de estados o directivas), que son relevantes para la función y rigen el comportamiento del sistema (pe., en la sociedad ciertas prácticas como la cooperación o sinergia). A diferencia de las entidades naturales, los artefactos materiales, como son sistemas artificiales, obedecen a reglas además de leyes. Se puede afirmar que las prácticas tecnológicas producen el nivel social de la artificis, o el "entorno directo" de la sociedad (lo que no son los componentes humanos en el sistema).

La diferencia crucial entre lo metafísicamente dado (una montaña, un río) y cualquier objeto "hecho por el hombre": lo artefactual (institución, regla de conducta, rascacielos, virus, etc.), es que lo metafísicamente dado debe ser aceptado, no se puede cambiar, no puede ser verdadero o falso, simplemente es, mientras lo artefactual nunca se debe aceptar sin crítica, debe ser juzgado por el hombre y entonces aceptado o rechazado y cambiado cuando sea necesario. Es el hombre, con su poder de escogencia, o la facultad de la volición, quien determina la falsedad o verdad de sus juicios, ya sea que correspondan o se contradigan con los hechos de la realidad. Estos no son correctos o erróneos, son el estándar de lo correcto o erróneo por el cual el hombre (racional) juzga sus metas, valores y escogencias.

\section{DISEÑO.}

Es el eje principal de las prácticas tecnológicas, como proceso deliberado, imaginativo, creativo e inteligente que produce en especial el invento de un artefacto, con el objetivo de resolver un "problema práctico" con medios y metas, cumpliendo con restricciones naturales (pe., ecológicas) y sociales (pe., económicas).

El proceso de diseño usualmente es realizado, no individualmente, sino por un subsistema concreto (tecnosistema), cuyos componentes son tecnólogos y artefactos en combinación. Metódicamente consiste en la "elaboración conceptual" (invento) de sistemas conceptuales o modelos que representan al artefacto o al cambio posible propuesto, como planos, esquemas, programas, especificaciones, modelos teóricos, algoritmos, reglas, cálculos, experimentos (laboratorio o campo), estudio de restricciones (físicas, sociales, económicas, ambientales, etc.). Sus acciones se apoyan en el conocimiento científico y tecnológico coetáneo, existente o por desarrollar, teórico o empírico. El concepto de diseño es el eje principal de la práctica tecnológica o tecnológico-científica y es un concepto praxiológico básico.

Debe observarse que en la investigación científica, la rama experimental tiene un carácter tecnológico (diseño del experimento) con fines cognitivos. Los experimentos van más allá de la simple observación, pues son intervenciones en una realidad material específica, constituyéndose en un "puente" entre ciencia y tecnología, que evidencia la intensa relación recíproca entre las prácticas científicas y tecnológicas.

\section{EJES DE LAS PRÁCTICAS TECNOLÓGICAS.}

Las prácticas tecnológicas o lo que los tecnologistas hacen, contienen dos ejes básicos de su actividad: (i) diseño y (ii) dirección/administración/ acción.

Un resumen de las características de las actividades del diseño y de la dirección/acción, son las siguientes:

(i) Eje del diseño: se diseñan (inventan y perfeccionan) los artefactos de alguna clase, 
sean naturales, artificiales o conceptuales, con base en conocimiento sustantivo (teorías sobre cosas materiales o sus procesos) o $\mathrm{co}$ nocimiento formal (lógica, matemáticas);

(ii) Eje de dirección/administración/acción: se dirigen y controlan la ejecución de los proyectos correspondientes, basados en conocimiento operativo (teorías o reglas sobre la estructura y organización de un sistema, o las situaciones problemáticas en las actividades humanas); es un tipo de administración. La "acción directa o empírica" (no administrativa o de dirección), la realizan solo algunas disciplinas tecnológicas, como la praxis médica o medicina 6 (pe., un cirujano, un terapeuta físico) y la educación (pe., un maestro o profesor), las cuales no solo dirigen o controlan las acciones, sino que las ejecutan directamente o mediante algún artefacto, sobre el referente de transformación, la primera sobre el cuerpo humano y la segunda en especial sobre la mente humana, pero en este caso básicamente mediante la transmisión de información.

A diferencia de las ciencias, sean fundamentales (básicas) o tecnológicas, las cuales resuelven "problemas cognitivos", la tecnología es una disciplina orientada a resolver problemas "prácticos", dirigidos como se ha descrito a transformar la realidad, con la invención, la mejora, el perfeccionamiento o el mantenimiento de artefactos o sistema de ellos (pe. una fábrica). La tarea cumple con las restricciones naturales, y con las demandas y especificaciones del cliente (ésta usualmente consiste del output y el presupuesto). Cada disciplina tiene sus características propias, dependientes del referente de transformación y su nivel óntico ${ }^{7}$, del tipo de problemática, de los objetivos, etc., pero todas cumplen con la definición general.

\section{TÉCNICA.}

Es una actividad o disciplina empírica, que consiste en la utilización de técnicas para manipular, diagnosticar o reparar algún artefacto o sistema de ellos (pe. computadoras). Es una actividad subsidiaria de la tecnología.
Algunas de ésas disciplinas son pe.: la microscopía, electrofisiología, cromatografía, imágenes por resonancia magnética, computación y control automáticos, en general dependientes del conocimiento científico-tecnológico.

Históricamente la tecnología fue pura técnica o principalmente empírica, hoy día hay que diferenciar la tecnología de la técnica y de la seudo-tecnología. El artefacto producido puede ser efectivo para su función, pero ineficiente debido su alto costo (económico o energético), como sucede con la seudo-tecnología, la cual es ineficiente debido a que ignora la determinación del mecanismo del artefacto, lo que se refleja en el alto costo de la técnica necesaria para su reparación y mantenimiento. El conocimiento científico que requiere la tecnología, tiene características particulares que se describirán a continuación.

\section{CIENCIA APLICADA ${ }^{8}$.}

Es un subconjunto del conocimiento científico el cual consiste de: (i) teorías particulares sustantivas y seguras con referente material (físico, químico, biológico, social, artefactual), elementos empíricos y reglas (científicas, técnicas y empíricas); (ii) teorías operativas (referentes: los procesos materiales como los de un computador) y metodológicas (referentes: las disciplinas). También se denominan: conocimiento científicotecnológico ("ciencias tecnológicas o de la ingeniería, medicina, educación, etc.").

Este campo disciplinario de la ciencia es importante, pues sirve de puente entre las prácticas científicas, el cual no siempre es considerado en el análisis de los conceptos de ciencia y tecnología. A veces se ha entendido la tecnología como ciencia aplicada, lo que es insuficiente pues olvida el componente básico de las disciplinas tecnológicas (prácticas sociales), como es el conocimiento tecnológico-científico (diseño, administración: dirección, control, acción), ya tratado. La ciencia aplicada o el conocimiento científicotecnológico, es solo uno de los componentes de la terna aquí definida. 
Una breve descripción de lo que le concierne a los componentes de la ciencia tecnológica, es la siguiente:

(i) A las teorías sustantivas les concierne la dilucidación de los mecanismos (procesos esenciales) y las leyes particulares y reglas, que rigen los sistemas materiales, sean naturales o sociales. Ejemplos pueden ser, en ingeniería clásica: la ley constitutiva de un material, o la teoría del vuelo de un avión, o el movimiento de un fluido particular; en medicina: la biología humana, en economía: ley del mercado, etc.

(ii) A las teorías o modelos operativas, que son sistemas conceptuales, les conciernen los "cambios de estado de artefactos existentes o por diseñar: si el referente es un sistema social, su organización (pe., las actividades en un aeropuerto), reglas de acciones humanas (pe. para el diseño), las relaciones hombre-máquina (organización de una fábrica), o si el referente es un sistema concreto su estructura (pe., una máquina). Si la máquina, es una computadora digital, el software diseñado es el "artefacto conceptual" que guía su secuencia de estados.

(iii) A las teorías metodológicas les concierne la metódica de investigación de un problema, es decir, en el que su referente es la disciplina misma (pe., el planeamiento de la investigación o la búsqueda de un diseño experimental apropiado para probar la teoría).

Los procesos del diseño requieren, más que teorías generales y exactas, la elaboración de teorías particulares y seguras, considerando los "grados de indeterminación" de sus modelos y datos, o sea, el conocimiento científicotecnológico o ciencia aplicada ("ciencias de la ingeniería o de la tecnología") coetáneo o existente. Para el ingeniero o tecnólogo, este conocimiento es un puente o elemento conceptual intermedio, necesario para el diseño y alcanzar la meta práctica. Así pe., la investigación biomédica produce conocimientos biológicos particulares (biología humana), que son la base del "diseño de tratamientos" (rama de la biotecnología).

En consecuencia es evidente, como se anotó anteriormente, que el concepto de tecnología no debe confundirse con el de ciencia aplicada ("ciencias de la ingeniería"), la cual constituye el vínculo recíproco entre el conocimiento dado por la ciencia básica y las práctica tecnológico- científicas (diseño), desde que busca conocimiento nuevo con potencial práctico y además "investiga a los artefactos mismos" (una característica nueva de la ciencia). Explícitamente, desde el punto de vista epistemológico, se tiene la terna: [ciencia básica $\cap$ ciencia tecnológica $\cap$ diseño].

La ciencia es relacionada a la tecnología como la teoría a la práctica, la segunda sin ciencia es imposible, la primera sin tecnología es inútil.

\section{TIPOS DE PREDICCIÓN.}

\section{Hay diferencias en las características de la predicción que hacen las ciencias fácticas básicas y las ciencias tecnológicas (o ciencias de la ingeniería).}

En las ciencias, sea la predicción científica (o retrodicción) o la predicción científico-tecnológica, consisten de: (a) enunciados legales (teorías científicas y tecnológicas y reglas del diseño) y datos empíricos y (b) enunciados de tendencias (extrapolación de las circunstancias presentes), siendo resultado de la aplicación de las teorías científicas (generales o particulares).

Las respuestas de una predicción científica permiten alcanzar un nuevo conocimiento o sirven de contrastación (verificación, refutación) de las teorías y de guía para la "acción". La particularidad y diferencia que tiene la predicción científica-tecnológica, es que "pronostica el posible comportamiento del artefacto diseñado". La falla o no de un diseño, permite el control y la seguridad de las reglas del diseño, de los datos empíricos utilizados (pe., las características de un sismo), y de la seguridad de los modelos producidos para representar el artefacto, lo que implica considerar su grado de indeterminación.

También la experimentación tecnológica, tiene la particularidad de que estudia el prototipo diseñado mediante modelos materiales del mismo (pe. un automóvil, un canal abierto, etc.). Sin embargo lo anterior no siempre es posible, pues hay campos tecnológicos que no pueden experimentar con prototipos de laboratorio, por su alto costo (pe., un puente en ingeniería estructural, un edificio sujeto a un sismo, una organización social, etc.). En estos casos la prueba concreta de los modelos usados y sus datos, es el comportamiento 
real ante las acciones a que el artefacto ha sido sometido (pe., una estructura bajo un sismo).

Laforma lógica de los enunciados predictivos es la misma para ambos tipos de predicción, pues se basa en enunciados nomológicos del tipo: [si la cosa $x$ está en el estado e' en el instante $t$, entonces la cosa $x$ estará en el estado $e^{\text {” }}$ en el instante $t "$ ], o [si $E$ sucede en el instante $t$ ' entonces $F$ sucederá en el instante $t$ " con probabilidad $p$.

De manera sintética se puede resumir así: "si \{Teoría científica, Datos\} resulta un enunciado singular"; se dice que esta conclusión es una predicción científica. Es evidente que ambos tipos, no coinciden con el concepto de expectativa subjetiva, conjetura, profecía, prognosis o predicción de sentido común.

\section{PREDICCIÓN CIENTÍFICAY CIENTÍFICO-TECNOLÓGICA.}

\section{Los tipos de pronosticación o predicción que hacen las ciencias (prácticas científicas y científico-tecnológicas) y las tecnologías (prácticas tecnológico-científicas) tienen una importante diferencia.}

La razón de la diferencia o particularidad, se debe a que a las prácticas tecnológicas no les basta con aplicar un resultado científico basado en una predicción científica o científico-tecnológica (aunque sean su fundamento), pues al contrario de ellas, las prácticas tecnológicas (diseño, ejecución) tienen que prever racionalmente, las consecuencias inmediatas y mediatas que pueden tener sus acciones transformadoras y sus objetivos deseados. Así, si la prueba experimental de una hipótesis o de una teoría científica, demuestra que no hay contrastación empírica con los hechos de la realidad, entonces la teoría o parte de ella se desecha sin consecuencias directas en las personas. Sin embargo, si una planta nuclear o un puente fallan, la situación social es enteramente diferente ahí que sus enunciados sean diferentes a los de la predicción científica, teniendo la forma siguiente: [si la cosa x está en el estado e' en el instante t', es necesario hacer K para que x alcance el estado deseado e", desigual a e [en el instante t"].
Entonces la previsión tecnológica es una predicción activa pues se basa en enunciados nomopragmáticos y entonces en reglas que se refieren a operaciones humanas: [si hay que alcanzar Z en el instante t" con probabilidad $p$, entonces hay que hacer $K$ en el instante $\left.t^{\prime}\right]$; las reglas o normas deberán estar basadas en el conocimiento científico, o sea, en alguna teoría sustantiva u operativa social, lo cual no siempre se tiene en forma elaborada. Primero se da el objetivo y luego la relación "medios-fin", siendo que los "medios" se podrían cumplir mediante un sistema de acciones de un sistema social concreto o de un tecnosistema, sin asegurar su efectividad.

\section{TIPOS DE PROBLEMAS.}

Un problema, para la ciencia o la tecnología como disciplinas, se presenta cuando hay una ausencia de conocimiento o alguna necesidad práctica, en algún campo. Sin embargo, los problemas tecnológicos, sean de cualquier tipo, son muy diferentes a los de las disciplinas científicas, pues son típicamente de diseño (invención, innovación), de acción, mejora (perfeccionamiento) y de mantenimiento, y no de descubrir alguna cosa o de probar una conjetura.

Hay dos tipos de problemas científicos y tecnológicos:

(i) Los conceptuales, como la lógica y las matemáticas, que solo requieren de herramientas conceptuales para solucionarlos;

(ii) Los empíricos: como los tecnológicos (diseño), que además requieren conocimiento tecnológico y de considerar las necesidades, restricciones y especificaciones naturales o sociales. Los problemas llamados "prácticos" solo demandan conocimiento técnico, ordinario y artesanal.

También existe otra partición del conjunto de problemas que trata la tecnología, denominados directos e inversos, que con mayor frecuencia se presentan a los diseñadores. Son los siguientes: 
(i) Los problemas tecnológicos directos, se refieren al mejoramiento o perfeccionamiento de un artefacto o proceso existente, son bien definidos y en general se podría decir que tienen soluciones únicas (pe.: "dado un artefacto existente con defectos de diseño y operación, perfeccionarlo o corregirlo", "dada una edificación con daños estructurales, corregirlos o repararlos" o "dadas las intenciones y circunstancias de una persona pronosticar su comportamiento".

(ii) Los problemas tecnológicos inversos requieren innovación radical: diseñar (inventar) un nuevo artefacto o encontrarle un nuevo uso a alguno, cumpliendo con determinadas especificaciones, como la función o el comportamiento esperado, pero sin especificar a los materiales o los procesos, que son respectivamente seleccionados o definidos en el proceso del diseño. Por eso la función puede concebirse y realizarse con varias alternativas para los mecanismos (procesos en el sistema). Por tanto, en general, son problemas impropiamente planteados y típicamente no tienen soluciones únicas, pues son muchas. Ejemplos pueden ser: "diseñar (inventar) un puente, sujeto a acciones externas (pe., un sismo) y condiciones y restricciones naturales"; "diseñar (inventar) un motor de plasma para que funcione en vuelos espaciales" (F. Chang D.); diseñar (inventar) una empresa o firma productiva", "diseñar (inventar) un sistema de control de la calidad de la educación" (SINAES). Estos ejemplos son casos típicos de la tecnología o ingeniería inver$s a$, pues pueden tener muchas soluciones, que dependen del estado del arte, del tipo de medios (materiales o conceptuales), de la estructura seleccionada, recursos disponibles (equipo, presupuesto) y de las metas propuestas.

Para redundar, otros ejemplos de problemas de este tipo, son los siguientes: "dado un comportamiento deseado de algo, imaginar cómo se podría obtener"; "dado el output de un sistema concreto, encontrar el input"; "dado el comportamiento de una persona adivinar sus intenciones"; "dada la falla de una edificación, develar sus causas"; "dado el estado de un enfermo, diagnosticar su causa". Como se señaló, la característica particular, es que a diferencia con los problemas directos, estos pueden tener muchas soluciones.

\section{MARCO TEÓRICO GENERAL.}

\section{El concepto general de tecnología descrito en párrafos anteriores, se puede representar por medio de la terna o modelo conceptual siguiente: Tecnología: \{práctica social, conocimiento, artefacto\}.}

Este marco da las herramientas para la comprensión del fenómeno tecnológico de nuestro tiempo. Los tres significados de los elementos de la terna están intrínsecamente vinculados y forman la estructura básica del marco, son genéricos e importantes para intentar capturar todo el fenómeno. La definición es general, implicando una ampliación o extensión del concepto "clásico" de las "ingeniería duras", a acciones de transformación nuevas que incluyen referentes naturales o sociales y conceptuales.

Cuando se dice tecnología (concepto), se está incluyendo a los tres elementos de la terna, a saber: el (i) son "actividades orientadas" (procesos o cambios de estado en individuos y cosas) en algún subsistema social que realiza el diseño y dirección; el (ii) son el resultado del diseño: "sistemas conceptuales" que modelan o se refieren a (i) (actividad) o/y al (iii) (artefacto), o representan el conocimiento (ciencia tecnológica) necesario para el proceso del diseño y para la explicación del mismo artefacto; el (iii) es un sistema, sea material (cosa) o conceptual (idea), producto de (i) (actividad) en base al (ii) (conocimiento). En el artefacto está cristalizada, tanto la acción como el conocimiento, de ahí la natural reducción social del concepto de tecnología al artefacto. Éste además, se convierte en un elemento social por su valor de uso, si pierde este valor, queda fuera de este mundo social, se convierte en un objeto material más (o en un simple desecho).

Es importante observar el hecho, ya dilucidado, que para "apropiarse" de un tipo de tecnología, es necesario conocer, respecto a todo 
artefacto o sistema de artefactos dados, los elementos del "modelo mínimo del sistema (social en este caso)", a saber: [(i) componentes (seres humanos); (ii) ambiente o entorno (lo que no son los componentes); (iii) estructura: conjunto de relaciones (vínculos o conexiones) entre componentes9 y entre éstas con el entorno, representadas por la organización; (iv) mecanismo: procesos esenciales que hacen realidad la función y (v) superestructura: reglas o leyes que rigen el comportamiento (cambio) de sus partes y de la totalidad.

$\mathrm{Si}$ de un artefacto se conociera solo su input y output (caja negra) la tecnología sería dependiente, lo contrario solo se alcanza dilucidando al sistema, es decir, es necesario convertir la caja negra en traslúcida. Es por lo mismo, que es necesaria la comprensión del fenómeno tecnológico.

\section{CLASIFICACIÓN DE TECNOLOGÍAS.}

\section{Cada disciplina es caracterizada por su referente de transformación o control y por su objetivo deseado.}

A continuación se da una lista provisional de tecnologías, en forma extensional y en función de su referente y objetivo. No se pretende agotar la lista, solo se tiene por objeto ilustrar en lo posible el tema.

(A) Referente material: (i) tecnología física: ingeniería eléctrica, electrónica, nuclear, civil, arquitectura, mecánica, industrial, nanotecnología, la informática (información y su procesamiento por medios automáticos), ingeniería de computadoras, tecnología de la información, inteligencia artificial o el diseño de procesadores de información y robots, computación, ingeniería aeronáutica, ingeniería espacial, ingeniería nuclear, etc.; (ii) tecnología química, orgánica e inorgánica: ingeniería química, química industrial, etc.; tecnología bioquímica: farmacología, bromatología, etc.; (iii) tecnología biológica o biotecnología: agronomía, medicina (terapia o el diseño de tratamientos), veterinaria, tecnología de alimentos, ingeniería genética, ingeniería biomecánica, etc.; (iv) tecnología biosocial: medicina social (epidemiología descriptiva y normativa, psicología social), psicología experimental aplicada, psiquiatría, terapia cognitiva-conductual, etc.; (v) tecnología social o sociotecnología: medicina social, trabajo social, la sociología y la politología aplicadas o "ciencias políticas", arquitectura, urbanismo: planeamiento de ciudades, tecnologías de la economía (planeación económica, ciencia de la administración, macroeconomía normativa), jurisprudencia, investigación de operaciones; educación, legislación, finanzas y política pública en general. Obviamente esta lista no contiene todas las especialidades, ni todas las interdisciplinas ${ }^{10}$, actuales o posibles.

(B) Referente conceptual: (i) tecnología física (objetual o "dura"): "ingeniería" del software (diseño del software para computadores físicos digitales, etc.), programación funcional, programación lógica, funcionales lógicos y programación, inteligencia artificial, sistemas expertos, programación genética, lingüística computacional, redes neuronales, etc.; (ii) tecnología filosófica, epistémica: praxiología (estudio de la acción humana), tecnoética (estudio de los códigos morales relacionados con la tecnología).

(C) Sin referente: tecnologías generales: no tienen referentes fácticos, se concentran en los aspectos estructurales (son portátiles), son teorías de sistemas: teorías de redes, información, autómatas, control, optimización, etc.

La clasificación anterior no es exhaustiva y tiene obviamente un carácter provisional, dado el carácter histórico del desarrollo científicotecnológico. La mayoría de las prácticas tecnológicas, por las exigencias técnicas de un "encargo", requieren la cooperación organizada de especialistas pertenecientes a varios campos de la división de trabajo. En estas circunstancias, es que se puede decir que existe una actividad interdisciplinaria, la cual aparece como lo contrario de la división de trabajo, dada a su integración como una empresa colectiva. Esto sucede también en la investigación científica.

Las definiciones usuales que las entidades académicas o profesionales hacen de cada disciplina, las describen en sus particularidades y 
ramas, pero todas caben en la definición general. Además, como se puede observar, muchas de las disciplinas clasificadas anteriormente para efectos de ilustración, no corresponden a las "ingenierías duras" o "clásicas" y en el pasado no eran consideradas como ingeniería o tecnología, aunque desde un marco general y moderno si lo son.

Por otra parte, se hace necesario estudiar simultáneamente y desde diferentes aspectos la sociedad y la naturaleza. Esta tarea impone no solo organizar los conocimientos por materias en el plano disciplinario (como la clasificación anterior), sino también por problemas, en el plano interdisciplinario. Claro está que estas formas de organización de los conocimientos están intervinculadas: en el proceso de solución de los problemas prácticos, la organización del saber por materias debe transformarse en la organización por problemas, para que después, la organización por problemas pueda nuevamente transformarse en la organización por materias.

La definición general que aquí se ha dado abarca, en primera instancia, a todas las disciplinas de transformación de la realidad, es decir, es una necesaria ampliación del concepto de tecnología. Estas nuevas concepciones son producto de la revolución científica-tecnológica (RCT) producida en la segunda mitad de SXX, en la que los procesos científicos y tecnológicos se amplían, vinculan e interactúan cada vez más, convirtiéndose en un factor de producción indispensable.

\section{FILOSOFÍA DE LA TECNOLOGÍA.}

\section{El estudio ontológico, epistemológico y ético de la tecnología se llama filosofía de la tecnología o tecno-filosofía.}

El desarrollo de una tecno-filosofía es relativamente nuevo, posiblemente como reacción social sobre la necesidad de controlar las acciones tecnológicas sobre la naturaleza. Esta rama de la ciencia filosófica se especializa en el estudio de cada disciplina o campo de conocimiento. Por ejemplo la iatrofilosofía o filosofía de la medicina: la rama de la epistemología que trata los supuestos filosóficos de las ideas y prácticas médicas que surgen en la actividad profesional e investigativa.

Los problemas propios de la tecno-filosofía tratan la dilucidación general de los conceptos básicos: ciencia y tecnología, su diferencia y relación, artefacto y su nivel óntico, leyes y reglas tecnológicas, dependencia, ética, presuposiciones filosóficas.

Así, en el caso de la ética social, se presenta la pregunta: ¿es "mala" la tecnología para la sociedad? Una respuesta puede ser la siguiente: como las prácticas tecnológicas, sean metódicas o en los fines y funciones de sus productos (artefactos o cambios tecnológicos), no son ideológicamente neutrales, pues son dependientes de intereses y decisiones políticas, como por ejemplo: “alcanzar la explotación máxima de los recursos naturales y humanos al menor costo y sin importar las consecuencias", la tecnología podría calificarse como buena, diabólica o ambivalente, dependiendo por tanto de los valores éticos y morales existentes o considerados. Así se está de acuerdo en que la bomba atómica y la producción de polución, son diabólicas por su falta de humanidad o por su daño del ambiente de la sociedad. Estos hechos, ha tenido consecuencias ideológicas, como son la tecnofilia y la tecnofobia, que exhiben algunos movimientos sociales.

Es decir, la tecnología como actividad social solo tiene una autonomía relativa. Por otra parte, el conocimiento científico-tecnológico alcanzado históricamente por las ciencias básicas y tecnológicas, es un conocimiento objetivo (verdades relativas históricamente, pero concretas), independiente de la dirección de los objetivos de las prácticas mismas, lo cual hace en este aspecto, triviales las objeciones anti-tecnología.

\section{OBSERVACIONESYCONCLUSIONES.}

Se ha visto que las palabras técnica, tecnología, ingeniería, recubren varias de las diferencias que hay entre las actividades del técnico, ingeniero, médico, educador, etc., frente a las realidades de los problemas técnicos que dominan hoy ramas de las ciencias naturales. En este "resumen" se han trazado algunas líneas de demarcación, evidencia- 
do los obstáculos epistemológicos existentes. Se considera que su interpretación permitiría orientar las acciones, en especial educativas, en el sentido "correcto" al dilucidar y separar las falsas ideas (lo ideológico) en este campo de la cultura.

Es un hecho que la mayor parte del público en general y hasta filósofos, científicos o tecnólogos, usualmente confunden a la tecnología con los artefactos (un caso muy corriente se da con las "computadoras", las que además se sabe no solo computan). Como se ha tratado, la tecnología coincide con el artefacto, solo en el sentido de que los hace, no porque los descubre o encuentre. Sin embargo, se piensa que esta falsa idea o confusión epistemológica, es producto de un efecto psicológico y social, resultado del hecho de que en el artefacto se cristaliza la acción y el conocimiento humano social (la duración).

Las nociones desarrolladas son de gran utilidad para efectos de la educación (en todos los niveles), como es el diseño de los perfiles profesionales y programas de estudio y que son evidentemente necesarias para no confundir, las capacidades y habilidades necesarias en cada disciplina (pe., ingenieros o tecnólogos, con otras disciplinas científicas como las matemáticas o las ciencias fácticas). $\mathrm{O}$ como sucede muy corrientemente, con la denominación errónea de una carrera o un título (pe., ingenieros tecnólogos ${ }^{11}$, tecnología agronómica, ingeniería en biotecnología, etc.), o cuando para cumplir con alguna definición clásica de ingeniería, se le agregan al plan de estudio algunos cursos de ciencias básicas (pe., física, química, matemáticas, etc.). Esta idea en parte se debe a que muchas definiciones (ver diccionarios) de ingenierías particulares, afirman que son el resultado de la "aplicación" de la física, química, geología, etc., es decir, ciencias básicas, dejando por fuera lo esencial, las ciencias de la ingeniería (pe., mecánica del sólido, de fluidos, termodinámica, pedadogía, etc.).

Es un hecho que los profesionales en tecnología, en su función de tecnólogos o de profesores, usualmente no tratan el carácter de su práctica en forma general, lo que si debe ser obligación de los expertos en estos campos. También es usual que muchos especialistas en educación, no conocen las características generales de las prácticas cuyo currículo académico hay que diseñar. Igual sucede cuando se trata de diseño de la organización universitaria de sus disciplinas académicas o culturales y otras.

Además, estas nociones permiten comprender la diferencia que hay en el carácter de los distintos tipos de investigación y su enseñanza-aprendizaje, asunto en el cual existe a veces gran confusión para su planeamiento e interpretación educativa. Así en las tecnologías, como se ha descrito, la investigación tecnológica desarrolla el conocimiento científico-tecnológico (ciencia aplicada) y el tecnológico-científico (metódica del diseño y acción) que tienen características particulares en cada campo (pe., la ingeniería civil tiene referentes macro, la electrónica micro).

Otro hecho importante para la educación, es que si bien las ciencias existen en el currículo de la enseñanza en los niveles no universitarios, el aspecto tecnológico no es tratado y si se llega a considerar, se hace la mayor de las veces con las confusiones señaladas en estas notas. Además cuando esto se piensa, es con una noción lineal ciencia-tecnología (la idea de que la segunda surge de la primera), cuando en la realidad son procesos simultáneos e interrelacionados. Corregir esto es difícil, en parte por la falta de investigación educativa en estas líneas y de profesores preparados para ello.

Con la intención de llenar el vacío conceptual y programático en la enseñanza de las ciencias y tecnologías, han surgido movimientos con estos objetivos, denominados de "alfabetización científico-tecnológica", objetivo para el cual es necesario el estudio epistemológico previo de las características del conocimiento científico y tecnológico y su producción. Esto es imprescindible para definir las políticas de desarrollo de la ciencia y la tecnología, así como para el diseño y política de las instituciones de educación superior en un país.

\section{NOTAS.}

1. Esta nota introductoria es obtenida de Herrera 1990.

2. Ver gráficos adjuntos.

3. Se puede usar "práctica" o "praxis". 
4. Se entiende por "control" a la regulación, espontánea o deliberada, de una variable de un sistema, como en "control automático"; es un concepto clave de la cibernética, biología, ingeniería y ciencia de la administración.

5. Un sistema es un objeto complejo íntegro, en el que cada parte o componente, está relacionada por lo menos a otra componente. Se distinguen dos sistemas básicos: materiales (pe. un organismo) y conceptuales (pe., compuesto de constructos o conceptos, proposiciones, teorías). A su vez, los materiales son sistemas naturales, sociales o artifíciales (hechos por el hombre).

6. La medicina, como "ciencia aplicada" y tecnología para el cuidado de la salud, está compuesta de cuatro diferentes esferas, que interactúan e interceptan: investigación biomédica, o biología humana (ciencia tecnológica médica), terapia, o el diseño de tratamientos, una rama de la biotecnología (tecnología médica), medicina social (epidemiología descriptiva y normativa) y la praxis médica (práctica tecnológica: control, ejecución)

7. En ontología un nivel integrativo o de organización de la realidad, es una colección de entidades materiales (concretas), que comparten ciertas propiedades y leyes. La hipótesis más simple respecto a estos niveles, que son una realidad (la colección de todos los objetos reales), es que está compuesta de cinco niveles principales: físico, químico, biológico, social, tecnológico o artefactual. Las entidades en un supranivel, están compuestas de entidades perteneciendo a niveles inferiores, y los superiores han emergido en el curso de tiempo, sea por asociación o por el desarrollo de los elementos del nivel inferior (pe. las reacciones químicas y la evolución biológica, son parangones de emergencia). El nivel mental no se incluye porque los entes conceptuales, no son cosas sino procesos cerebrales (cambios de estado del cerebro). Cualquier nivel se puede analizar en subniveles: micro-, meso-, macro-, mega-niveles .

8. Se considera que este nombre responde a la imagen originaria de "aplicación" como superposición-impresión, lo cual implica una dualidad de objetos (lo que es aplicado difiere de aquel sobre lo que se aplica), además de la instrumentalidad y exterioridad, propios de la tecnología. En realidad lo que hay es una relación de constitución, es decir, la ciencia o conocimiento básico que se aplica, es una parte integrante de la existencia del nuevo objeto de conocimiento. Esta es la ciencia (subconjunto de la ciencia en general) que el autor prefiere denominar: ciencia tecnológica o conocimiento científicotecnológico.

9. En especial aquellas relaciones que mantienen al sistema unido o su integridad. Las estructuras son propiedades de sistemas, no hay estructuras en sí mismas, es decir son conceptos no cosas.

10. Las interdisciplinas son las ciencias de transición entre niveles ónticos adyacentes (pe., la fisicoquímica, la bioquímica, psicofisiología, etc.) y por tanto a las correspondientes tecnologías.

11. Esta denominación fue hecha hace algunos años por el ITCR (Instituto Tecnológico de Costa Rica), como un artificio legal para incorporarse al CFIA (Colegio Federado de Arquitectos e Ingenieros de Costa Rica), mediante la creación del Colegio de Ingenieros Tecnólogos!

\section{BIBLIOGRAFÍA}

Herrera, R. (1989). "Technology and Society" Proceedings of the ASEE 1989. Annual Conference. Lincoln, Nebraska.

Herrera, R.(1989). "Relation between Continuing Engineering Education and Traditional Engineering Education. Presentado en The Fourth World Conference on Continuing Engineering Education. Beijing, China.

Herrera,R. (1990). Tecnología y Sociedad”. En Revista de Filosofía Universidad de Costa Rica, 23 (67/68), 77-84.

Herrera, R. (2006a). "Sistema y lo Sistémico en el Pensamiento Contemporáneo". En Ingeniería 17 (2): 37-52, ISSN: 1409-2441. Extraído originalmente de: http://cariari.ucr. ac.cr/ macalvoh/. 
Herrera, R. (2005). "Tecnología: una concepción general”. En Revista Filosofía Universidad de Costa Rica. 43 (109/110), 99-108.

Herrera, R. (2009). "La tecnociencia y la educación: la problemática de su integración”. En Revista Filosofía Univ. Costa Rica. 48 (120/121), 9-17.

\section{SOBRE EL AUTOR}

\section{Rodolfo Herrera J.}

Doctor en Ingeniería Civil. Profesor Emérito de la Universidad de Costa Rica. Miembro del Consejo del Postgrado en Ciencia Cognoscitiva de la Universidad de Costa Rica. 